\title{
A Case of Idiopathic Orbital Inflammation with Shallow Anterior Chamber and Choroidal Detachment
}

\author{
Yohei Sato ${ }^{a, b}$ Natsuko Mano ${ }^{a, b}$ Hiroko Watanabe ${ }^{a, b}$ \\ Makiko Miyamoto $^{a, b} \quad K^{2}$ azuhiro Shimizu, ${ }^{a, b}$ Noriko Mukai ${ }^{b}$

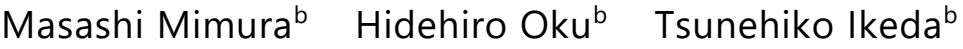 \\ ${ }^{\text {aD }}$ epartment of Ophthalmology, Takatsuki Hospital, Takatsuki City, Japan; bepartment of \\ Ophthalmology, Osaka Medical College, Takatsuki City, Japan
}

\section{Keywords}

Idiopathic orbital inflammation - Shallow anterior chamber - Choroidal detachment

\begin{abstract}
We report a case of idiopathic orbital inflammation with a shallow anterior chamber and choroidal detachments. This study involved an 87-year-old female patient who presented at our department after becoming aware of the progression of diplopia. Examination of the patient's right eye revealed proptosis, as well as conjunctival edema with dilated and tortuous blood vessels. The right-eye anterior chamber was shallow, and fundus examination revealed marked choroidal detachments. Magnetic resonance imaging revealed enlargement of the right-orbit extraocular muscles and a suspected compression of the right-orbit superior and inferior ophthalmic veins, yet no expansion of the cavernous sinus. We diagnosed the patient as having idiopathic orbital inflammation in her right orbit, and subsequently started corticosteroid therapy. One week after initiating treatment, the anterior chamber was found to be nearing a normal depth, and the choroidal detachments were found to have disappeared. Our findings revealed that the inflammatory swelling of the extraocular muscle due to idiopathic orbital inflammation resulted in compression of the right-orbit superior and inferior ophthalmic veins, thus leading to an apparent choroidal circulation disorder that presented with a shallow anterior chamber and marked choroidal detachments.




\section{Introduction}

Idiopathic orbital inflammation (IOI) is an idiopathic inflammatory condition of unknown cause that occurs in the orbital tissue, and it is classified as perioptic-type, periocular-type, apical-type, lacrimal-type, and diffuse-type orbital myositis, depending on the site at which it occurs in the orbit [1,2]. Reportedly, orbital myositis is associated with certain ophthalmic complications, such as optic neuropathy, retinal vein dilation, conjunctival vasodilation, nonischemic retinal vein occlusion, and central artery and vein occlusion [3, 4]. In this study, we report a case of IOI that developed with proptosis, ocular movement disorder, and conjunctival vasodilation, and was found to have a shallow anterior chamber and marked choroidal detachments, which rapidly improved with corticosteroid therapy.

\section{Case Presentation}

This study involved an 87-year-old female patient with a medical history of hypertension who was being followed up at the Department of Ophthalmology at Takatsuki Hospital, Takatsuki City, Osaka, Japan, 7 months before initial presentation due to paralysis of her left upper oblique muscle. An orbit magnetic resonance imaging (MRI) examination performed at the first visit revealed no obvious abnormality in the brain. The patient presented at our department after becoming aware of diplopia that was becoming worse. Upon examination, the patient was found to have proptosis, ocular movement limitation (especially, abduction disorder), and conjunctival edema with dilated and tortuous blood vessels in her right eye. We suspected carotid-cavernous sinus fistula (CCF), and thus subsequently referred her to the department of neurosurgery for a more detailed examination. An MRI examination revealed enlargement of the right-orbit extraocular muscles, yet no expansion of the cavernous sinus. Thus, she was suspected to have a retinal detachment in her right eye and was subsequently referred back to our clinic.

\section{Initial Findings}

In the patient's right and left eyes, her corrected visual acuity was 0.7 and 1.0 , and her intraocular pressure was 14 and $12 \mathrm{~mm} \mathrm{Hg}$, respectively. In the right eye, we observed proptosis, conjunctival edema with dilated and tortuous blood vessels, and a shallow anterior chamber, although with pseudophakia, yet no inflammation in the anterior chamber and vitreous cavity. Funduscopy imaging of the patient's right eye revealed retinal vein dilation and marked choroidal detachments in all quadrants (Fig. 1). No abnormalities were observed in her left eye. We used three-dimensional anterior segment optical coherence tomography(CASIA ${ }^{\circledR}$; Tomey Corporation, Nagoya, Japan) to measure the anterior chamber depth (ACD) and anterior chamber volume (ACV). In the patient's right eye, the ACD was $2.53 \mathrm{~mm}$, and the ACV was $88 \mathrm{~mm}^{3}$ (Fig. 2a). In her left eye, the ACD was $3.48 \mathrm{~mm}$, and the ACV was 167 $\mathrm{mm}^{3}$ (Fig. 2b). The coronal short-tau inversion-recovery (STIR) sequence of the MRI examination showed thickening of the right-orbit extraocular muscles, especially the lateral rectus and inferior rectus (Fig. 3). Moreover, although expansion of the superior ocular vein was observed, there was no abnormality of the vascular network and no expansion of the cavernous sinus. A general blood examination revealed a $4.3 \times 10^{3} / \mu \mathrm{L}$ white blood cell count and 0.08 $\mathrm{mg} / \mathrm{dL}$ C-reactive protein (CRP), yet negative results for thyroid antibody, soluble interleukin2 receptor antibody, IgG4, and collagen disease. 


\section{Treatment Progress}

We diagnosed the patient as having right-orbit IOI and initiated treatment with oral prednisolone (30 mg per day). One week after initiating treatment, the visual acuity in her right eye was found to have improved to 1.0. Furthermore, the proptosis had improved, the anterior chamber was becoming a normal depth, and the choroidal detachments were found to have disappeared. Moreover, the ACD and ACV were found to have increased to 3.74 and $178 \mathrm{~mm}^{3}$, respectively (Fig. 2c). One month after the initial examination, MRI findings revealed improvement of the thickening extraocular muscle. Two months after the initiation of the corticosteroid therapy, the patient's ocular movement disorder improved, and the diplopia disappeared. The dose of prednisolone was gradually tapered down, and there was no recurrence of inflammation.

\section{Discussion}

IOI is a rare inflammatory condition of unknown cause, and the inflammation occurs in areas of the orbital tissues such as the lacrimal gland, the eye globe, the extraocular muscles, and the optic nerve. IOI is characterized by sudden onset, and has various symptoms such as periorbital pain, swelling, diplopia, proptosis, ocular movement disorder, ptosis, and conjunctival congestion, etc. [1,2]. IOI may present as an acute type, a chronic type, and an atypical type, and it is known to have a differential diagnosis of CCF, thyroid eye disease (TED), malignant lymphoma (ML), IgG4-related ophthalmic disease (IgG4-ROD), and orbital cellulitis (OC). In this present case, we initially suspected CCF due to the symptoms of proptosis, conjunctival edema with dilated and tortuous blood vessels, and ocular movement disorder. However, the MRI examination performed at the department of neurosurgery revealed expansion of the superior ocular vein, yet no abnormality of the vascular network and no expansion of the cavernous sinus. Thus, the patient was deemed negative for CCF. The patient was also deemed negative for CCF due to the fact that the corticosteroid therapy was very effective.

Reportedly, IgG4-ROD is a disease in which tumors, enlargements, or hypertrophic lesions are in tissues other than the lacrimal gland, the trigeminal nerve, and the extraocular muscles [5]. In our present case, we observed swelling of the extraocular muscles, yet no abnormality was found around the lacrimal gland and the trigeminal nerve. Moreover, and although no biopsy of tissues such as the lacrimal gland was performed, the serum IgG4 was in the normal range, and IgG4-ROD was negative. Although a biopsy is required for a definitive diagnosis of ML, the serum soluble interleukin receptor (sIL-2R) antibody level is also useful for the diagnosis of ML. Our patient was deemed negative for ML, since the serum soluble interleukin receptor antibody level was within the normal range, and the treatment course was favorable. In TED cases, ocular movement disorder usually occurs in the direction in which the inflamed muscle antagonizes. In our case, all extraocular muscles were swollen, yet especially in the external muscle and inferior muscle. Ocular movement disorder was recognized in all directions, yet we deemed our patient negative for TED because the most obvious indicator was the abductive disorder. Nishikawa et al. [6] reported that CRP may be useful to differentiate IOI and OC. Our patient was deemed negative for OC because the general blood test revealed no inflammation (white blood cell count and CRP levels remained within the normal limits), there was no inflammation in the paranasal sinuses, and improvement was made only via the corticosteroid therapy without any treatment with antibiotics.

The present case had no periorbital pain, yet proptosis, ocular movement disorder, and conjunctival congestion were observed. MRI examination revealed the enlargement of 
extraocular muscles, and especially coronal STIR showed hyperintensity around the extraocular muscles; i.e., findings that are consistent with IOI.

It should be noted that the characteristic point of this present case is that the IOI was associated with a shallow anterior chamber and choroidal detachments. The reported causes of choroidal detachment include the following:

- $\quad$ choroidal inflammation secondary to perforating ocular trauma, retinal photocoagulation, cryotherapy, Vogt-Koyanagi-Harada disease, sympathetic ophthalmia, and scleritis [7];

- hypotony due to suture insufficiency after intraocular surgery, overfiltration after glaucoma surgery, and cilia body detachment after trauma [8];

- choroidal circulation disorder due to CCF, and compression of the vortex vein after scleral buckling surgery [9];

- choroid infiltration of a malignant tumor such as metastatic choroidal tumor, choroidal malignant melanoma, leukemia, and ML [10];

- $\quad$ inflammation through the site of perforation of the vortex vein after OC [11].

In 1993, Kurtz et al. [12] reported a case of orbital pseudotumor presenting as acute glaucoma with choroidal and retinal detachment. Moreover, Bernardino et al. [13] reported three cases of angle-closure glaucoma in association with orbital pseudotumor, and that the mechanism of angle-closure was anterior rotation of the ciliary body secondary to choroidal effusions resulting from the orbital inflammation. Diseases with choroidal inflammation, such as Vogt-Koyanagi-Harada disease and scleritis, can lead to the development of a shallow anterior chamber. Yet, in the present case there was no inflammation in the anterior chamber and vitreous cavity. Thus, we determined that the choroidal detachment was not caused by choroidal inflammation. Kwon and Lee [14] reported that kissing choroidal detachment and retinal vein occlusion occurred in a patient with IOI. In that study, they reported that orbital inflammation and edema may compress orbital structures and increase resistance in retinal veins, thus leading to vein congestion and occlusion, and therefore, elevated venous pressure may increase colloid leakage into the suprachoroidal space, and increased vascular permeability and decreased venous outflow may cause choroidal detachment. In our present case, first, swelling of the extraocular muscles occurred due to IOI, which then led to compression of the upper and lower eye veins, ultimately resulting in choroidal circulation disorder. Moreover, marked choroidal detachments occurred as a result of forward movement of the ciliary body and the shallow anterior chamber. Corticosteroid therapy improved the swelling of the extraocular muscles, relieved the compression of the ocular veins, and improved the choroidal circulation. As a result, the marked choroidal detachments disappeared, and the ACD was becoming normal.

In conclusion, the findings in this present case indicate that IOI may cause various eye symptoms such as CCF, so strict attention is required. 
Acknowledgement

The authors wish to thank John Bush for editing the manuscript.

\section{Statement of Ethics}

This case study was approved by the Ethics Committee of Osaka Medical College.

\section{Disclosure Statement}

There are no conflicts of interest to report for all authors.

\section{Funding Sources}

Not applicable.

\section{Author Contributions}

Y. Sato, N. Mano, N. Mukai, and K. Shimizu: equal contribution to patient management, conception of the paper, data analysis and interpretation, manuscript drafting, and literature search. H. Watanabe, M. Miyamoto, M. Mimura, H. Oku, and T. Ikeda: co-writing of the manuscript, literature search. Y. Sato: design of the paper, co-writing of the manuscript, literature search, manuscript editing and revision, as well as final approval. All authors read and approved the final manuscript.

\section{References}

1 Rootman J. Why "orbital pseudotumour" is no longer a useful concept. Br J Ophthalmol. 1998 Apr;82(4):33940.

2 Kubota T. Orbital myositis. In: Gran JT, editor. Idiopathic Inflammatory Myopathies - Recent Developments. InTech; 2011. pp. 123-42. Available from: http://www.intechopen.com/articles/show/title/orbitalmyositis.

3 Foroozan R. Combined central retinal artery and vein occlusion from orbital inflammatory pseudotumour. Clin Exp Ophthalmol. 2004 Aug;32(4):435-7.

4 Swamy BN, McCluskey P, Nemet A, Crouch R, Martin P, Benger R, et al. Idiopathic orbital inflammatory syndrome: clinical features and treatment outcomes. Br J Ophthalmol. 2007 Dec;91(12):1667-70.

5 Goto H, Takahira M, Azumi A; Japanese Study Group for IgG4-Related Ophthalmic Disease. Diagnostic criteria for IgG4-related ophthalmic disease. Jpn J Ophthalmol. 2015 Jan;59(1):1-7.

6 Nishikawa Y, Oku H, Tonari M, Matsuo J, Sugasawa J, Ikeda T. C-reactive protein may be useful to differentiate idiopathic orbital inflammation and orbital cellulitis in cases with acute eyelid erythema and edema. Clin Ophthalmol. 2018 Jun;12:1149-53.

7 Yamamoto N, Naito K. Annular choroidal detachment in a patient with Vogt-Koyanagi-Harada disease. Graefes Arch Clin Exp Ophthalmol. 2004 Apr;242(4):355-8.

8 Bellows AR, Chylack LT Jr, Hutchinson BT. Choroidal detachment. Clinical manifestation, therapy and mechanism of formation. Ophthalmology. 1981 Nov;88(11):1107-15.

9 Schutz JS, Richoz O. Risk factors for development of choroidal detachment after scleral buckling procedure. Am J Ophthalmol. 2012 Jan;153(1):188-9.

10 Kase S, Suimon Y, Ishijima K, Ishida S. Rhegmatogenous retinal detachment in a patient with choroidal melanoma simulating choroidal detachment: a case report. J Med Case Reports. 2018 Dec;12(1):389. 


\section{Case Reports in Ophthalmology}

Sato et al: A Case of Idiopathic Orbital Inflammation with Shallow Anterior Chamber and Choroidal Detachment

11 Farhi P, Kurup S, Abdelghani WM. Orbital cellulitis associated with combined retinal and choroidal detachments. Eye (Lond). 2007 Jul;21(7):1009-10.

12 Kurtz S, Moisseiev J, Gutman I, Blumenthal M. Orbital pseudotumor presenting as acute glaucoma with choroidal and retinal detachment. Ger J Ophthalmol. 1993 Feb;2(1):61-2.

13 Bernardino CR, Davidson RS, Maus M, Spaeth GL. Angle-closure glaucoma in association with orbital pseudotumor. Ophthalmology. 2001 Sep;108(9):1603-6.

14 Kwon JM, Lee SJ. Kissing choroidal detachment in a patient with idiopathic orbital inflammatory syndrome. Korean J Ophthalmol. 2019 Aug;33(4):386-8.
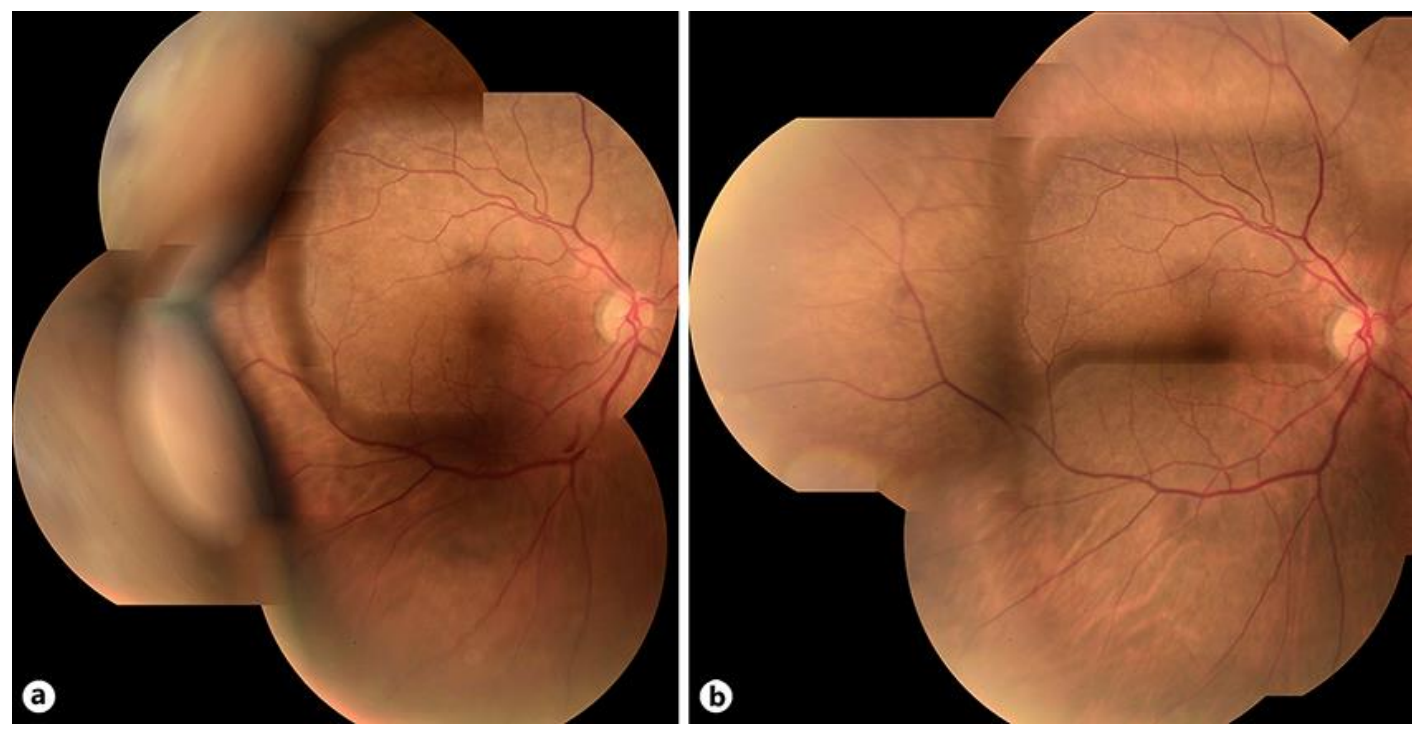

Fig. 1. Funduscopy images of the patient's right eye. Retinal vein dilation and marked choroidal detachments can be seen at the initial examination (a), but choroidal detachments disappeared 7 days after the start of corticosteroid therapy (b). 


\section{Case Reports in Ophthalmology}

Case Rep Ophthalmol 2020;11:8-15 DOI: $10.1159 / 000505314$

(c) 2020 The Author(s). Published by S. Karger AG, Basel www.karger.com/cop

Sato et al:: A Case of Idiopathic Orbital Inflammation with Shallow Anterior Chamber and Choroidal Detachment
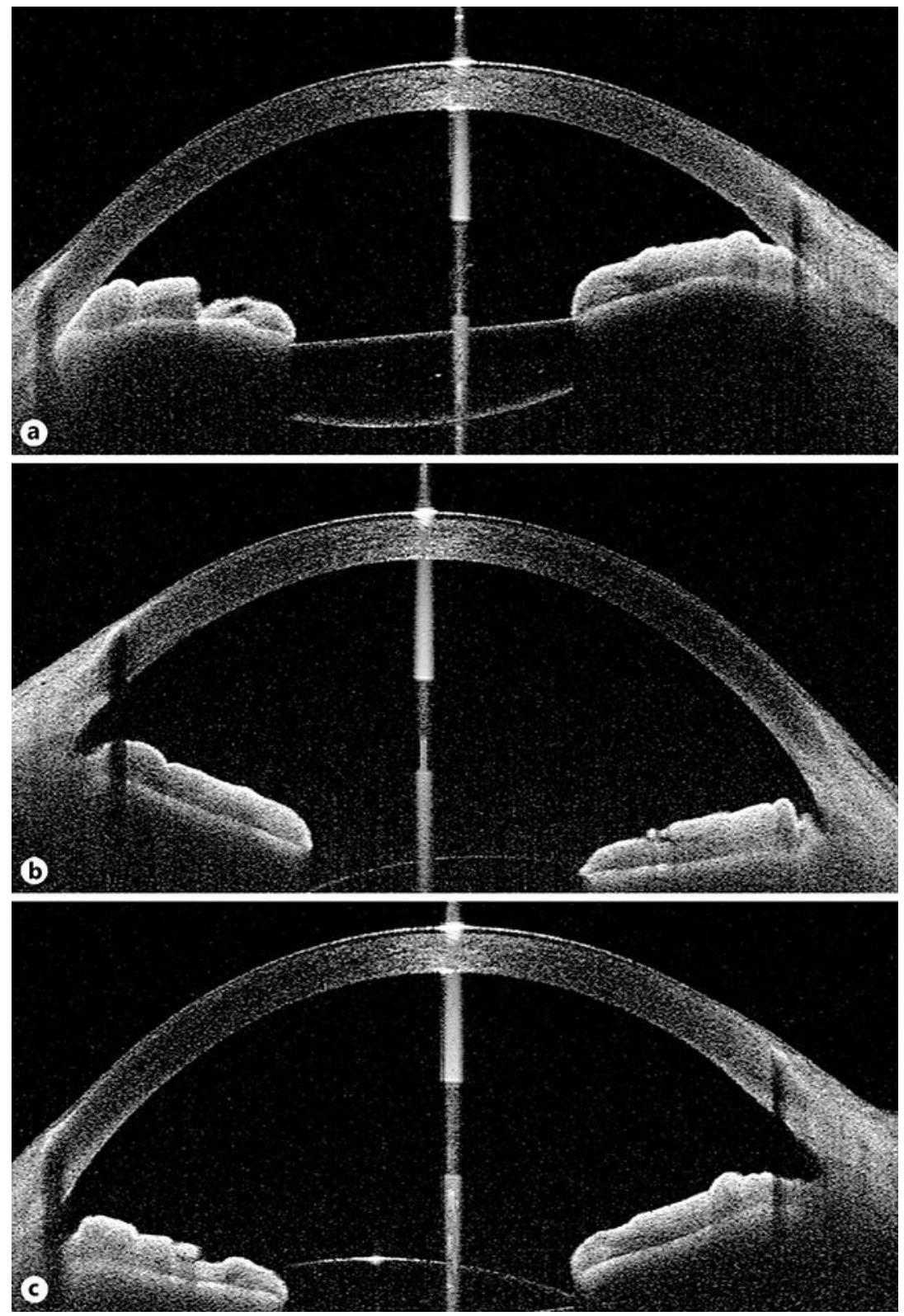

Fig. 2. Anterior segment optical coherence tomography images. The ACD was $2.53 \mathrm{~mm}$, and the ACV was $88 \mathrm{~mm}^{3}$ in the patient's right eye (a), although the ACD was $3.48 \mathrm{~mm}$ and the ACV was $167 \mathrm{~mm}^{3}$ in the left eye (b) before treatment. After treatment, ACD and ACV increased to 3.74 and $178 \mathrm{~mm}^{3}$, respectively (c). 


\section{Case Reports in Ophthalmology}
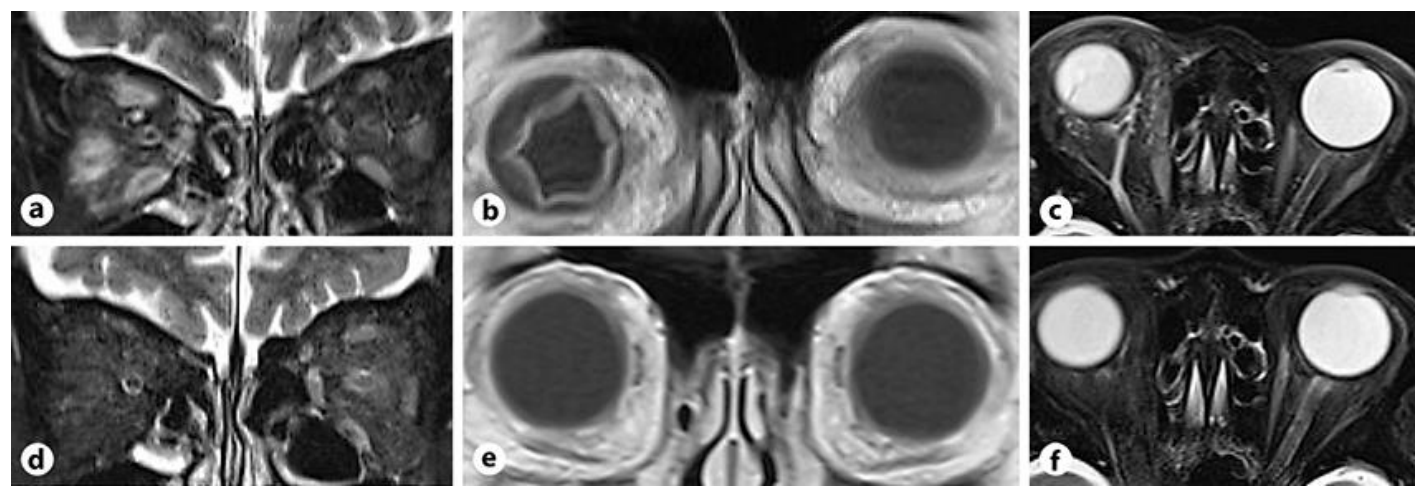

Fig. 3. Orbital MRI scans obtained before and after corticosteroid therapy. Coronal STIR (a), T1W1 (b), and axial STIR (c) MRI scans obtained before treatment. Coronal STIR (d), T1W1 (e), and axial STIR (f) MRI scans obtained after treatment. Enlargement of the right extraocular muscles, especially the lateral and inferior rectus (a), marked choroidal detachments of the right eye (b), and expansion of the superior ocular vein $(\mathbf{c})$, all showing complete regression after treatment $(\mathbf{d}-\mathbf{f})$. 